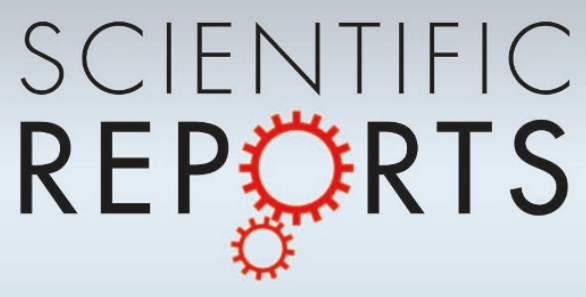

OPEN

SUBJECT AREAS:

NANOPARTICLES

RAMAN SPECTROSCOPY

NANOPHOTONICS AND

PLASMONICS

FLUORESCENCE SPECTROMETRY

Received

24 July 2013

Accepted

8 January 2014

Published

27 January 2014

Correspondence and requests for materials should be addressed to

G.W.L. Iguowei.lu@ pku.edu.cn) or Q.H.G. (qhgong@pku.edu.cn)

\section{Photoluminescence of a single complex plasmonic nanoparticle}

\author{
Tianyue Zhang' ', Guowei Lu', Hongming Shen' ', Kebin Shi', Yuanyuan Jiang ${ }^{2}$, Dongsheng Xu \\ \& Qihuang Gong'
}

\begin{abstract}
${ }^{1}$ State Key Laboratory for Mesoscopic Physics, Department of Physics, ${ }^{2}$ Beijing National Laboratory for Molecular Sciences, State Key Laboratory for Structural Chemistry of Unstable and Stable Species, College of Chemistry and Molecular Engineering, Peking University, Beijing 100871, China.
\end{abstract}

We report detailed investigations of the photoluminescence $(\mathrm{PL})$ generated from an individual gold nanoflower, a highly branched plasmonic nanoparticle. Compared to nanostructures with simple shapes, such as spheres, nanorods, and bipyramids, nanoflowers exhibit more distinct features, i.e., the PL spectra and far-field emission patterns are strongly dependent on the wavelength and polarization of the excitation light. The experimental results are qualitatively explained using theoretical calculations. In addition, the intrinsic PL signal is highly dominated by localized surface plasmon resonances. The crucial role of plasmonic coupling in complex nanostructures during the plasmon-enhanced PL process is highlighted. The findings contribute to a deeper understanding of the PL properties of metallic nanoparticles. This study will be beneficial for several potential applications, including optical imaging and sensing in the fields of materials science and biology.

N oble metallic nanoparticles support localized surface plasmon resonances (LSPRs), collective oscillations of surface electrons that provide an avenue for the control and manipulation of photons at the nanoscale, known as optical antennas ${ }^{1,2}$. Considerable interest has been focused on plasmonic nanoparticles with controllable three-dimensional structures for optimizing the manipulation of photons in various application $\mathrm{s}^{3-14}$. Anisotropic metal nanoparticles with complex shapes are fascinating because of their unique spectroscopic properties, which depend on the geometry and three-dimensional structures of these nanoparticles. For instance, multiple plasmon resonances, which result in polarization-dependent multipeak scattering, are extremely sensitive to the local dielectric environment ${ }^{3,4}$. In addition, experimental measurements and theoretical calculations have demonstrated a strong electromagnetic field enhancement near the surfaces of complex nanostructures ${ }^{5-7}$. The formation of three-dimensionally structured nanoparticles, such as dendrites, multipods, and highly branched nanoflowers or nanostars, is a fairly recent development ${ }^{5,6,8,9}$. Such complex-structured nanoparticles are potential candidates for a variety of applications, including optical probes ${ }^{10}$, active surface-enhanced Raman scattering (SERS) substrates ${ }^{7,8,11,12}$, biological and chemical sensors ${ }^{4,13}$, and near-infrared absorbers in disease therapy ${ }^{14}$.

To date, most experimental methods for investigating single complex-structured nanoparticles have been based on optical scattering, which arises directly from the coupling and interactions of plasmon modes with the incident light. Extensive studies have combined scattering measurements and theoretical modeling to reveal the size- and shape-dependent optical properties of such nanoparticles ${ }^{3,15-18}$. Other methods, for example, using SERS signals to perturbatively characterize complex plasmonic systems, have also been applied. Confocal Raman imaging has been utilized to determine the spatial distributions of hot spots in a single micrometer-scale flowerlike silver particle ${ }^{12}$. The wavelength-dependent angular distribution of a SERS imaging pattern has been reported to discriminate particle dimers from higher-order aggregates ${ }^{19}$. Recently, the intrinsic photoluminescence (PL) of metallic nanoparticles has received considerable attention. Although the quantum efficiency of the emission process is low (e.g., $\sim 10^{-4}$ for gold nanorods), this inefficiency can be compensated for by the large excitation cross sections at the plasmon resonances. Additionally, the PL of metal nanoparticles is free of photobleaching and photoblinking. Therefore, PL can be considered a better candidate than fluorescent molecules for optical labeling applications ${ }^{20,21}$. Single-photon and multi-photon excitation PL has been reported for various plasmonic nanoparticles, including nanospheres ${ }^{21-23}$, nanorods ${ }^{24-28}$, bipyramids ${ }^{29}$, nanocubes ${ }^{30}$, and nanoshells ${ }^{31}$. These studies shed light on the PL properties and mechanisms of metallic nanoparticles, which will benefit their future applications. However, the investigation of the PL properties of complex-structured nanoparticles, rather than those with simple shapes, is insufficient to date. 
In this study, we report detailed investigations of the PL characteristics of a single gold nanoflower (AuNF), a highly branch-structured plasmonic nanoparticle. The PL spectra and far-field emission patterns of single AuNFs exhibit more complex features than those of simple nanostructures, and the results reveal distinct features under different excitation wavelengths and a strong excitation polarization dependence. The PL spectra and emission patterns provide a complementary approach to the optical scattering method. Additionally, we observed SERS blinking accompanying the strong PL background from contaminated AuNFs, manifested as dynamic fluctuations in the SERS spectra and far-field emission patterns. The SERS blinking patterns indicated the activation of specific LSPR modes of the complex nanostructure. Theoretical calculations were performed to simulate the scattering spectra and emission patterns, and the results of these calculations agreed well with the experimental results.

\section{Results}

We synthesized the AuNFs according to a previously reported amino-reducing method $^{9}$, which provided a high yield of branched gold nanoparticles with abundant petal-shaped tips. Figure 1a presents typical transmission electron microscopy (TEM) images of the AuNFs synthesized as above. The inset shows a representative magnified gold nanoflower (AuNF). Morphologically, the nanoparticles are uniformly flower-like, consisting of a quasi-spherical solid core with many short irregular tips, with overall dimensions of $150 \pm$ $30 \mathrm{~nm}$. The tips have a variety of shapes and sizes; some of the tips grow longer and look like petals. The extinction spectrum exhibits a broad and well-defined surface plasmon resonance peak at a wavelength of $\sim 680 \mathrm{~nm}$, as shown in Figure 1b. The synthesized AuNFs were then immobilized on a glass substrate, and detailed characterizations of their optical properties were performed via single-particle spectroscopy. The single-particle measurements were based on a combined-detection configuration, which was described in detail in our previous study ${ }^{29}$. Most of the AuNFs were isolated and well separated on the glass coverslip so that single nanoparticles could be optically resolved. Figure 2 depicts the PL spectra of two representative individual AuNFs excited at wavelengths of $532 \mathrm{~nm}$ and $633 \mathrm{~nm}$ and the corresponding white-light total internal scattering spectra.

The scattering and PL spectra of individual AuNFs are distinctly varied compared with those of a colloid ensemble due to unavoidable deviations in size and shape. The size and shape of the AuNFs vary substantially from particle to particle. The geometric information regarding individual AuNFs was accessed via scanning electron microscopy (SEM). The model shapes of the AuNFs that were developed based on the SEM images were utilized in the theoretical calculations of the scattering spectra using the finite-difference timedomain (FDTD) method. For the AuNF (particle 1) shown in Figure 2a, which has a highly symmetric geometry, the modeled construct has a large number of tips distributed almost symmetrically around the spherical core surface (the diameter of the core is $150 \mathrm{~nm}$, and the ellipsoid-shaped tips range in length from 10 to $30 \mathrm{~nm}$ ). The
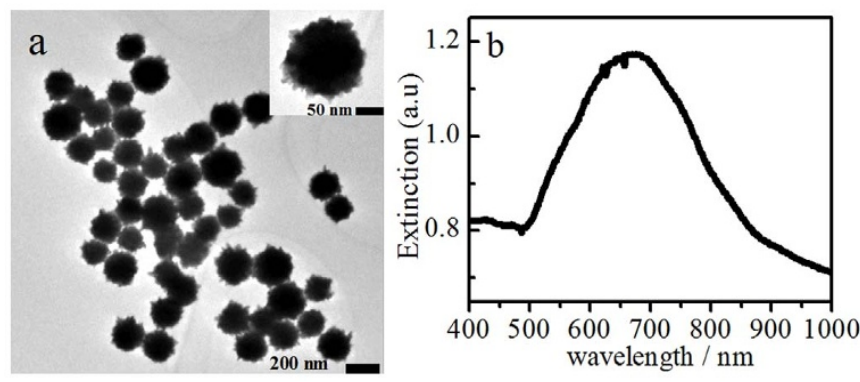

Figure $1 \mid$ Characterization of the gold nanoflowers synthesized in the study: (a) TEM images and (b) UV-visible spectrum. a
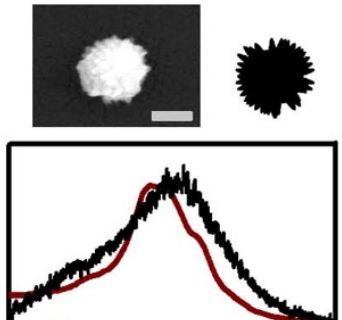

550600650700750800850

\section{$\mathrm{b}$}
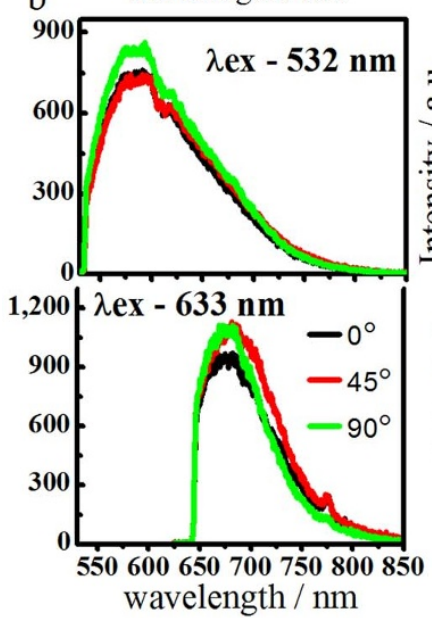

C
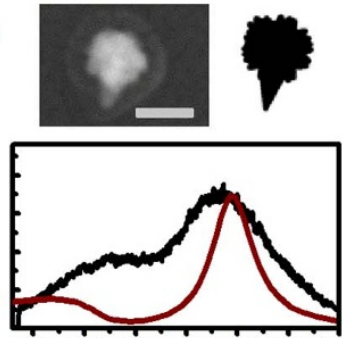
wavelength / nm
550600650700750800850
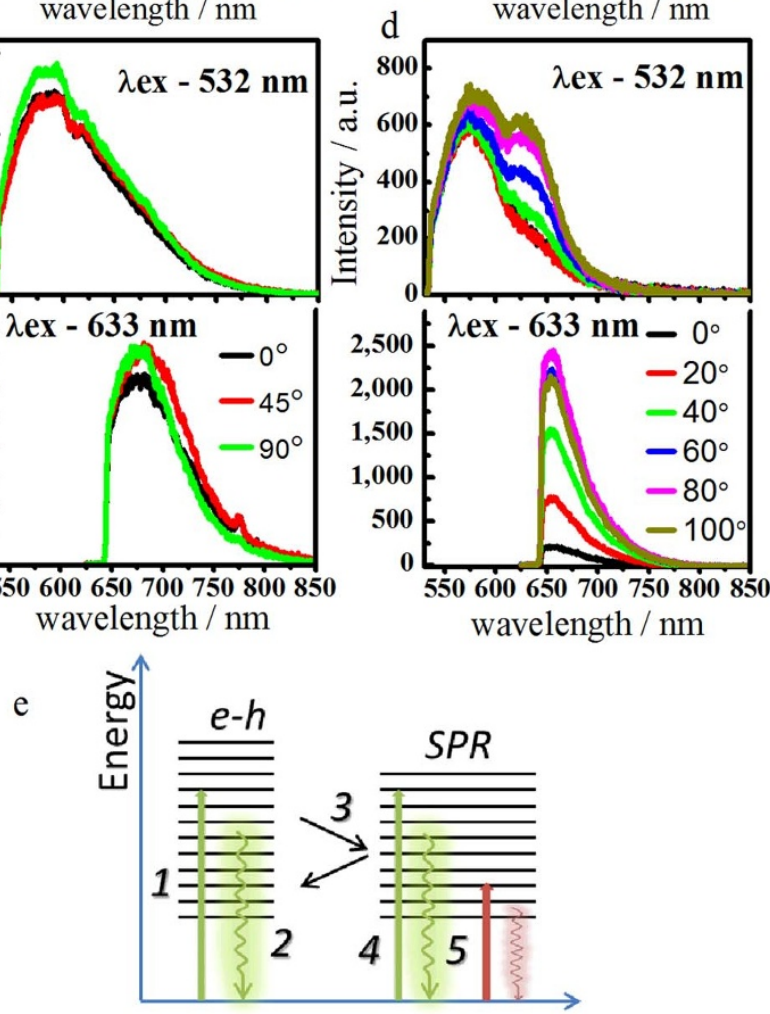

Figure $2 \mid$ Photoluminescence spectra of two representative AuNFs excited by a CW $532 \mathrm{~nm}$ laser and a $633 \mathrm{~nm}$ laser. (a) and (c) are the SEM images of the AuNFs and their modeled geometries for the numerical simulation; the scale bars in the SEM images represent $100 \mathrm{~nm}$. The experimental dark-field scattering of the AuNFs is plotted in black, while the theoretical simulation extinction spectra are shown in brown. (b) and (d) are the PL spectra of the corresponding AuNFs under excitation with different polarization angles. (e) Schematic diagram of radiative decay channels with different excitation wavelengths. The solid green lines represent excitation with the $532 \mathrm{~nm}$ laser, which excites both interband transitions by creating e-h pairs and the LSPR modes. The solid red line depicts the direct excitation of the LSPR with the $633 \mathrm{~nm}$ laser. The wavy lines represent emission from two decay channels.

complex hybridization of plasmons associated with the core and the manifold tips thus results in a broad scattering peak. The anisotropic AuNF (particle 2) shown in Figure 2c was modeled as a nanoflower with a very large tip consisting of a spherical core (with a diameter of $90 \mathrm{~nm}$ ), one large pseudo-conical tip (with a tip length of $35 \mathrm{~nm}$ and a tip-rounding radius of $5 \mathrm{~nm}$ ), and other small tips. According to the plasmon hybridization concept ${ }^{15}$, the observed broad plasmon resonances result from the interaction of plasmons associated with the core and the tips. For real AuNFs, there exist many small protruding tips around the core that contribute to the plasmon hybridization and lead to spectrum broadening. The scattering peak at $730 \mathrm{~nm}$ corresponds to the low-energy dipolar mode with plasmon oscillations confined around the large tip region ${ }^{6,16}$, while the 
plasmon band at $620 \mathrm{~nm}$ is attributed to the excitation of higherorder plasmon modes with the coupling of the core-tip and intertip modes ${ }^{15,16}$. Here, the calculated scattering spectrum is in qualitative agreement with the measured spectrum.

In previous extensive studies of nanospheres, nanorods, and nanobipyramids, the PL spectral profiles of particles with such simple geometries were quite similar to the particles' scattering spectra, and the observed PL spectra were independent of the excitation wavelength ${ }^{26-29}$. This wavelength independence can be attributed to the fact that simple-geometry nanoparticles (spheres or rods) have a single dominant LSPR mode in the emission band. For the complex and irregular AuNFs in the present experiments, the spectral features exhibit distinct behaviors under different excitation wavelengths, as illustrated in Figure 2. The PL peak is blue-shifted in comparison with the scattering peak because of the large number of available electrons near the initial energy of the laser excitation ${ }^{22,32}$. Specifically, the PL spectra excited by the $532 \mathrm{~nm}$ laser exhibit a greater blue shift but are less intense compared with those by the $633 \mathrm{~nm}$ laser when the excitation power of both lasers is the same at $40 \mu \mathrm{W}$. First, the excited electrons are usually pumped and populated at the initial energy states above Fermi level due to the laser excitation. Because of the fast damping rate of each LSPR mode $(\sim \text { femtosecond })^{33,34}$, radiative decay directly from the high-energy LSPR modes is possible. When the nanoparticles are excited at $532 \mathrm{~nm}$, both LSPR coupling and interband transitions can occur for the AuNFs, and these processes cannot be separated because of their spectral overlap. The results are analogous to the molecular luminescence arising directly from the higher vibronic levels of excited states, as shown in Figure 2e, where such radiative decay can be comparable to the rapid internal relaxation of a molecular system $^{35,36}$. Regarding the PL spectra resulting from excitation with the $633 \mathrm{~nm}$ laser, the LSPRs of the AuNFs are excited directly, and thus radiative decay occurs through plasmon emission. Thus, the PL spectra of AuNFs excited by the $633 \mathrm{~nm}$ laser present less blue shift compared with the spectra of AuNFs excited by the $532 \mathrm{~nm}$ laser. Second, with $532 \mathrm{~nm}$ excitation, the decay associated with the combination of photoexcited electron-hole pairs dramatically modifies the quantum efficiency of the AuNF emission. Moreover, in contrast with nanorods and nanobipyramids, the core-tip and tip-tip plasmonic modes of the AuNFs are strongly coupled and interacted; such strong coupling of the LSPR modes leads to significant near-field enhancement, accompanied by an increased non-radiative LSPR damping. The increase in non-radiative LSPR loss greatly reduces the PL efficiency; this phenomenon has been systematically demonstrated in dimer systems ${ }^{37}$. In addition, it has been experimentally demonstrated that the quantum yield is higher by approximately an order of magnitude for particles with a plasmon resonance greater than $650 \mathrm{~nm}$ relative to that for gold nanospheres with an LSPR less than $560 \mathrm{~nm}^{28}$. All of these factors should account for the higher PL intensity observed when the AuNFs are illuminated with a $633 \mathrm{~nm}$ laser than that observed with illumination by a $532 \mathrm{~nm}$ source.

The more interesting result is the polarization-dependent PL of individual AuNFs. The PL spectra were recorded with the excitation laser linearly polarized along various directions. Figure 2a illustrates a highly symmetric AuNF, which is representative of the shape of most of the AuNFs (according to the statistical shape distribution determined from the TEM images). The PL spectra of this nanoparticle exhibit less polarization dependence with either 532 or $633 \mathrm{~nm}$ excitation because the high-density tips are distributed uniformly around the core surface. For the PL excited by the 633-nm laser, the LSPRs of the AuNFs are excited directly and then undergo radiative decay through plasmon emission, while the PL elicited by the 532-nm laser presents considerable blue shift because of the strong LSPR coupling and interband transitions. For comparison, a representative AuNF with a highly anisotropic shape, as shown in Figure 2c, was investigated. The PL emission band near $670 \mathrm{~nm}$ strongly reflects the polarization-dependent feature of the excitation of the low-energy dipolar plasmon mode of this AuNF. In fact, this dipolar mode may be ascribed to the longitudinal plasmon resonance dominated by the large tip, according to the SEM image. Therefore, the intensity of the PL observed with excitation at $633 \mathrm{~nm}$ varies as the excitation polarization rotates and reaches a maximum when the polarization of the excitation is almost parallel with the long axis of the tip. When the AuNF is excited by cross-polarized incident light, the longitudinal plasmon mode cannot be excited efficiently, resulting in the weakest PL emission. In contrast, under illumination with the 532-nm laser, the PL spectra present two obvious bands. The peak near $570 \mathrm{~nm}$ exhibits almost polarization-independent features. Such a green emission band may originate from the coupled decay of short-wavelength radiative LSPR modes and the unpolarized interband transition enhanced by these plasmon resonances. However, the PL shoulder near $640 \mathrm{~nm}$ is highly polarization-sensitive, probably because of the long-wavelength LSPRs dominated by the large tip. In this study, our results imply that the excitation wavelength- and polarization-dependent PL of AuNFs is strongly related to the LSPRs and their coupling, which are jointly dependent on the geometrical complexity of the AuNFs and the interband transitions.

In the following, we selected two other representative AuNFs (particles 3 and 4), which have polarization-dependent spectra, to record in situ the angular distribution of the far-field PL radiation, i.e., the images of the intensity distribution in the back focal plane (also known as the Fourier plane). Such extremely strong intrinsic PL radiation has been utilized to determine the three-dimensional orientations of isolated individual gold nanorods and nanobipyramids ${ }^{29,38,39}$. Herein, the PL radiation pattern provided a complementary technique, in addition to the spectra, for characterizing the plasmonic emission features. The atomic force microscopy (AFM) images confirm that single AuNFs were measured rather than aggregates, as shown in the Figure 3 insets. Figure $3 \mathrm{c}$ and d presents the corresponding PL patterns for the two particles under excitation at $532 \mathrm{~nm}$ and $633 \mathrm{~nm}$ and at different linearly polarized angles. Similar to the case of a radiative dipole close to the interface, the PL emission of the AuNFs immobilized on the glass substrate flows primarily into the high-index medium, presenting a sharp maximum at the critical angle. Previous studies of gold nanospheres and nanorods have reported scattering or PL emission patterns with dipole-like properties $^{17,29,38,39}$. There are often two bright lobes distributed perpendicularly to the dipole moment in the imaging patterns. For a single nanosphere, the radiation pattern will rotate with the rotation of the excitation polarization because of the high symmetry of the spherical shape. In this case, the preferential resonance orientation is dependent on the excitation polarization. For nanorods (or nanobipyramids), in which the dipole moments of the longitudinal resonant mode are fixed along the longitudinal axis of the antenna, a welldefined pattern is presented regardless of the polarization of the incident light. In contrast, AuNFs that possess multiple plasmonic modes display explicit excitation polarization- and wavelengthdependent features in their PL radiation patterns. As illustrated in Figure 3, the PL imaging patterns observed with 633-nm illumination are highly sensitive to the angle of polarization due to the radiative properties of the LSPRs. Because of the highly heterogeneous tip orientations, different excitation polarizations can induce different core-tip coupling modes. All of these modes are related to the specific nanostructure configuration of the AuNFs. However, those induced by excitation at $532 \mathrm{~nm}$ exhibit nearly an entire bright ring. These results can be attributed to the dephasing or depolarization of the plasmons $s^{24,32}$ and the probable non-polarized interband transition ${ }^{28,32,37}$.

To understand the polarization-dependent PL emission, numerical simulations were performed to develop explanations. For simplicity, the nanostructure of the AuNFs was modeled as several 


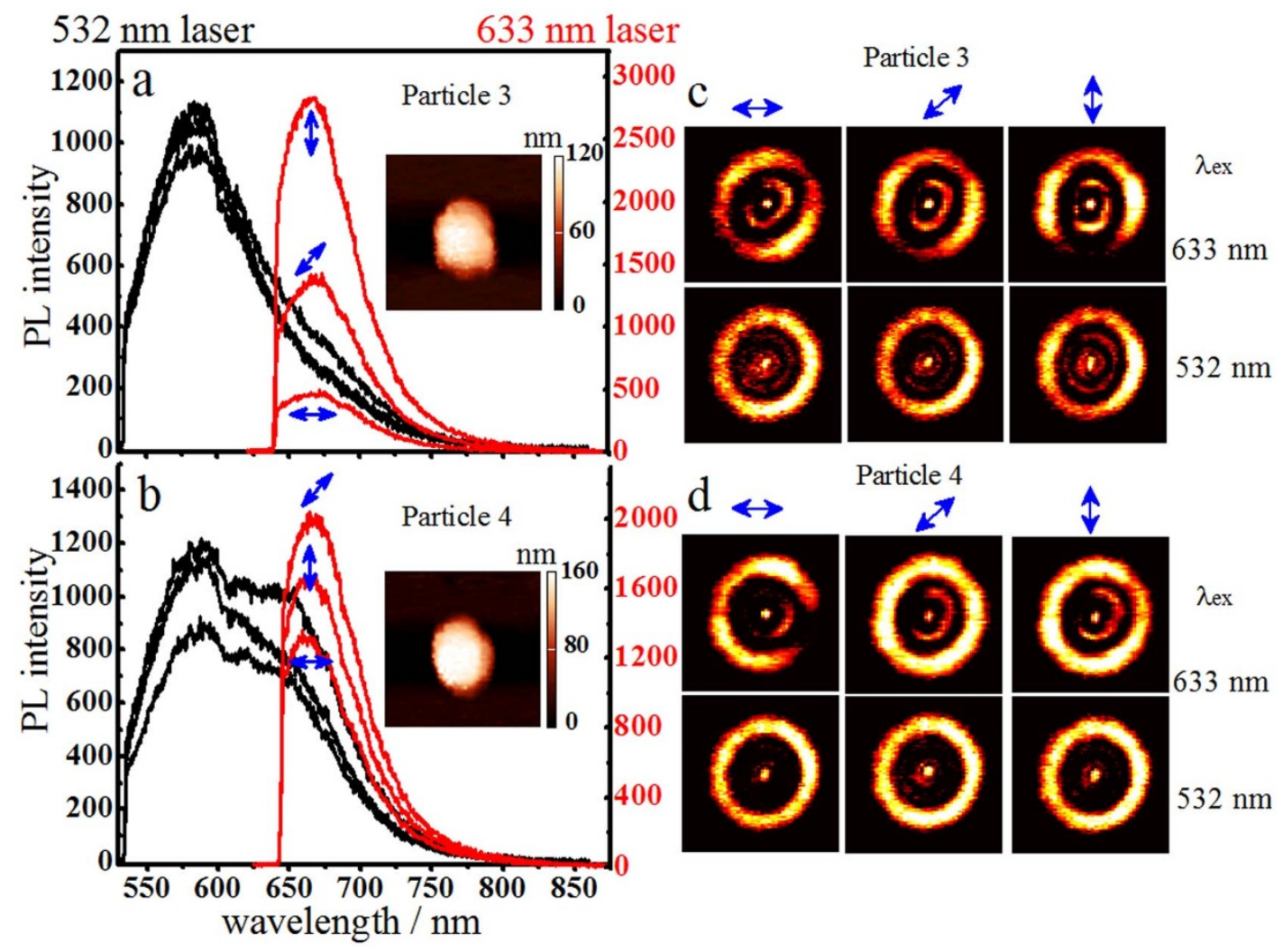

Figure $3 \mid$ (a), (b) Polarization dependence of the PL spectra of the AuNFs under excitation at wavelengths of $532 \mathrm{~nm}$ (black) and $633 \mathrm{~nm}$ (red) for two representative AuNFs. (c), (d) The PL imaging patterns of the same AuNFs under different incident-light polarizations. The blue arrows indicate the directions of the excitation polarizations.

ellipsoid-shaped tips around a spherical core. The far-field imaging patterns were calculated using the near-to-far-field transformation (NTFF) method after a single FDTD run with a monochromatic plane-wave excitation ${ }^{40}$. The investigation of the scattering light for a certain incidence angle and polarization illumination permits an efficient and accurate calculation of the far-field pattern, which is related to the PL imaging pattern at the corresponding wavelength. We calculated the radiation patterns at wavelengths of $633 \mathrm{~nm}$ and $730 \mathrm{~nm}$, corresponding to the two scattering peaks in the spectrum (Figure $4 \mathrm{~b}$ ). As for the plasmonic resonances confined to the AuNF core, the scattering patterns at a wavelength of $570 \mathrm{~nm}$ were also simulated for comparison. It can be clearly seen from Figure $4 \mathrm{c}$ that the AuNF structure exhibits different scattering responses for different incident wavelengths, and the patterns are polarization sensitive. The patterns elicited with excitation at $570 \mathrm{~nm}$ present radiative dipole features; the emission pattern exactly follows the illumination polarization. However, the patterns related to the long-wavelength mode of the AuNFs present distributions that are more complex; they are not dipole-like distributions, and the relationship between the pattern and the excitation polarization is not clear. All of these findings are, indeed, the intrinsic properties of the AuNFs, which are determined by the specific nanostructure and the plasmon coupling. It should be noted that, although the experimental imaging patterns present more uniform features, the calculated scattering response of the plasmonic resonances confined to the AuNF core exhibits behavior similar to that of a single dipole because the calculations cannot include the effects of the above-mentioned processes of plasmon depolarization and interband transitions.

Notably, we also observed Raman blinking accompanying a strong PL background for a few AuNFs due to the strong SERS enhancement. The Raman signals could be the result of the introduction of contaminants on the AuNF surfaces during sample preparation. The presence of the sharp tips of the AuNFs causes very large enhance- ments in the local field forming numerous "hot spots" on the individual nanoparticles. Such flower-like or star-shaped nanoparticles have been recently reported to serve as efficient SERS substrates with a high reproducibility ${ }^{8,9,11,12,41}$. The SERS experiments were performed under illumination with a 633-nm laser with circular polarization to excite the AuNFs. One hundred SERS patterns and the 50 correlated SERS spectra were recorded continuously with an integration time of $0.2 \mathrm{~s}$. The results are presented in the left-hand panel of Figure 5. Both the SERS spectra and the imaging patterns of the AuNFs fluctuate over time, which is an intrinsic SERS property of an extremely low concentration of Raman molecules. These few or single molecules may diffuse to different "hot spots" on the AuNFs at different moments in time, thus producing Raman blinking ${ }^{19,42}$. The stable background signal is from the PL emission of the AuNFs rather than from the fluorescence of the absorbed molecules, which is confirmed by the agreement of the background spectra with the PL spectra of the AuNFs in both peak position and spectral shape. The SERS emission imaging patterns recorded at different blinking moments of the same AuNF shown in Figure 5 represent the pure Raman blinking signal after subtracting the PL background imaging patterns. At the same time, the PL background pattern of the AuNF is also shown for comparison, i.e., the spectra and imaging patterns of the AuNF PL are stable under circularly polarized illumination and, therefore, are clearly distinct from the SERS blinking.

Localized coupling between the molecule and the antenna results in the modulation of the emission direction of the SERS signal. We illustrate this point by presenting theoretical calculations of the emission patterns of a radiative dipole (i.e., a point source) placed at various positions with various dipole-moment orientations with respect to the AuNF surface, as shown in Figure $5 \mathrm{c}$. The point emitter couples locally to the antenna plasmon modes; then, the active plasmon modes can couple with other plasmon modes. All of these excited plasmon modes in turn couple with the radiation field and 

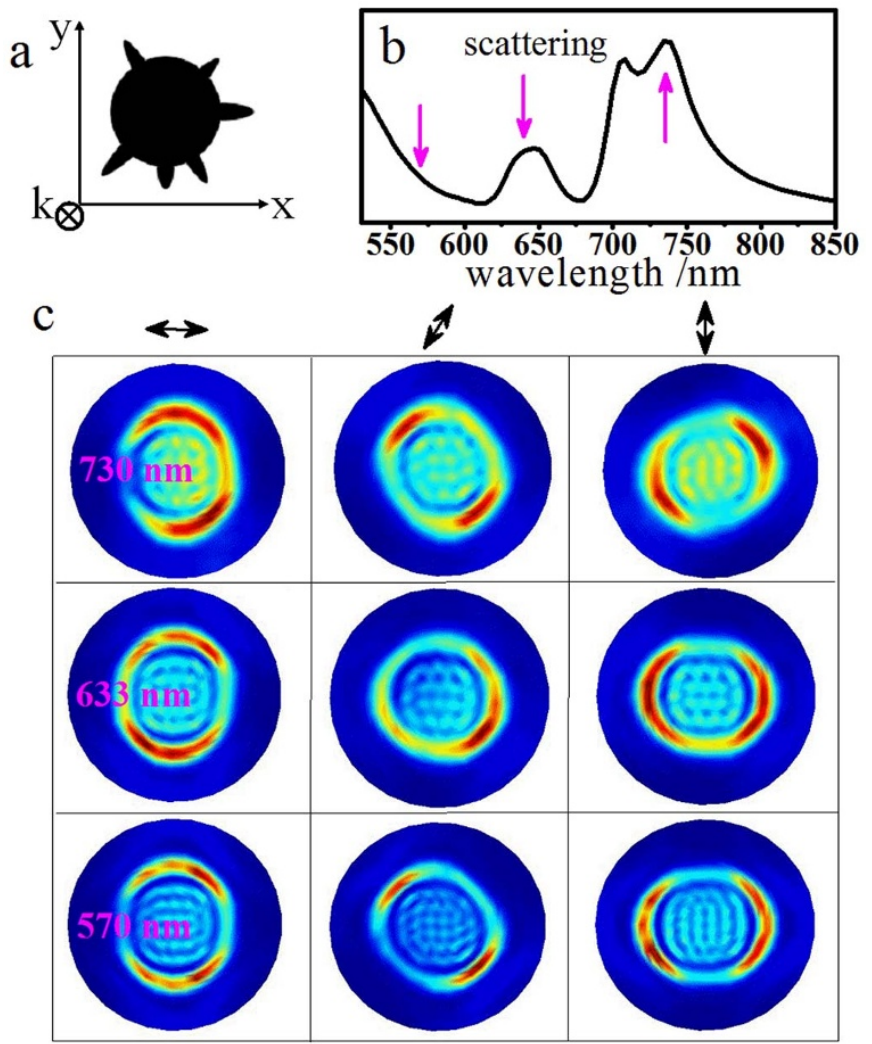

Figure 4 | Theoretical simulations of wavelength- and polarizationdependent emission patterns of a single modeled AuNF. The radiation patterns at wavelengths of $633 \mathrm{~nm}$ and $730 \mathrm{~nm}$ correspond to the two scattering peaks related to the tip modes in the spectrum. Scattering patterns at a wavelength of $570 \mathrm{~nm}$, which corresponds to the plasmonic resonances related to the AuNF core, are also presented. The black arrows indicate the directions of the excitation polarizations. may cause interference, which eventually determines the angular emission in the far field ${ }^{43-45}$. As displayed in the right-hand panel of Figure 5, the radiation patterns can be divided into two classes: patterns $a, b$, and c correspond to when the emitter coupled to the right horizontal tip of the AuNF, while patterns d, e and f correspond to when the emitter was near the bottom vertical tip. These patterns demonstrate the strong redirection of the dipole emission caused by the antenna mode regardless of the molecular orientation. In the present SERS blinking experiments, the SERS imaging patterns vary over time. This variation can be attributed to the extremely low molecule concentration, such that the plasmon modes of the AuNF are excited locally, partially, and occasionally, resulting in fluctuations in the SERS patterns. In contrast, the PL emission patterns, which can be regarded as the radiation of all the excited antenna modes of the AuNF under plane-wave illumination, present stable features when the illumination polarization is fixed.

\section{Discussion}

The PL from bulk gold was first reported by Mooradian, who studied smooth gold film and attributed the PL to the radiative recombination of the excited electrons in the sp band with d-band holes ${ }^{46}$. Afterwards, PL was studied to be considerably more efficient in gold films with rough surfaces because of the plasmon enhancement of the radiative transitions ${ }^{47}$. To date, the origin of the PL of gold nanoparticles remains an open question, and two mechanisms have been proposed: plasmon-enhanced interband transitions ${ }^{22,24,25,30,48}$ and radiative decay from excited particle plasmons (LSPs) ${ }^{23,26,27,29,32}$. The LSPRs in plasmonic nanoparticles offer an additional decay channel, which can compete with the direct recombination of excited electrons with holes. Extensive studies have been reported regarding particle plasmon emission, which gives rise to PL and has proven to be much more efficient than the direct photon emission via e-h recombination $^{23,27,28,37}$.

Regarding the origin of the PL of gold nanoparticles, as pointed out by Orrit and colleagues, regardless of the decay pathway from which the PL emission originates, the PL is primarily determined by the plasmon dipole moment. Therefore, observations of the plasmon

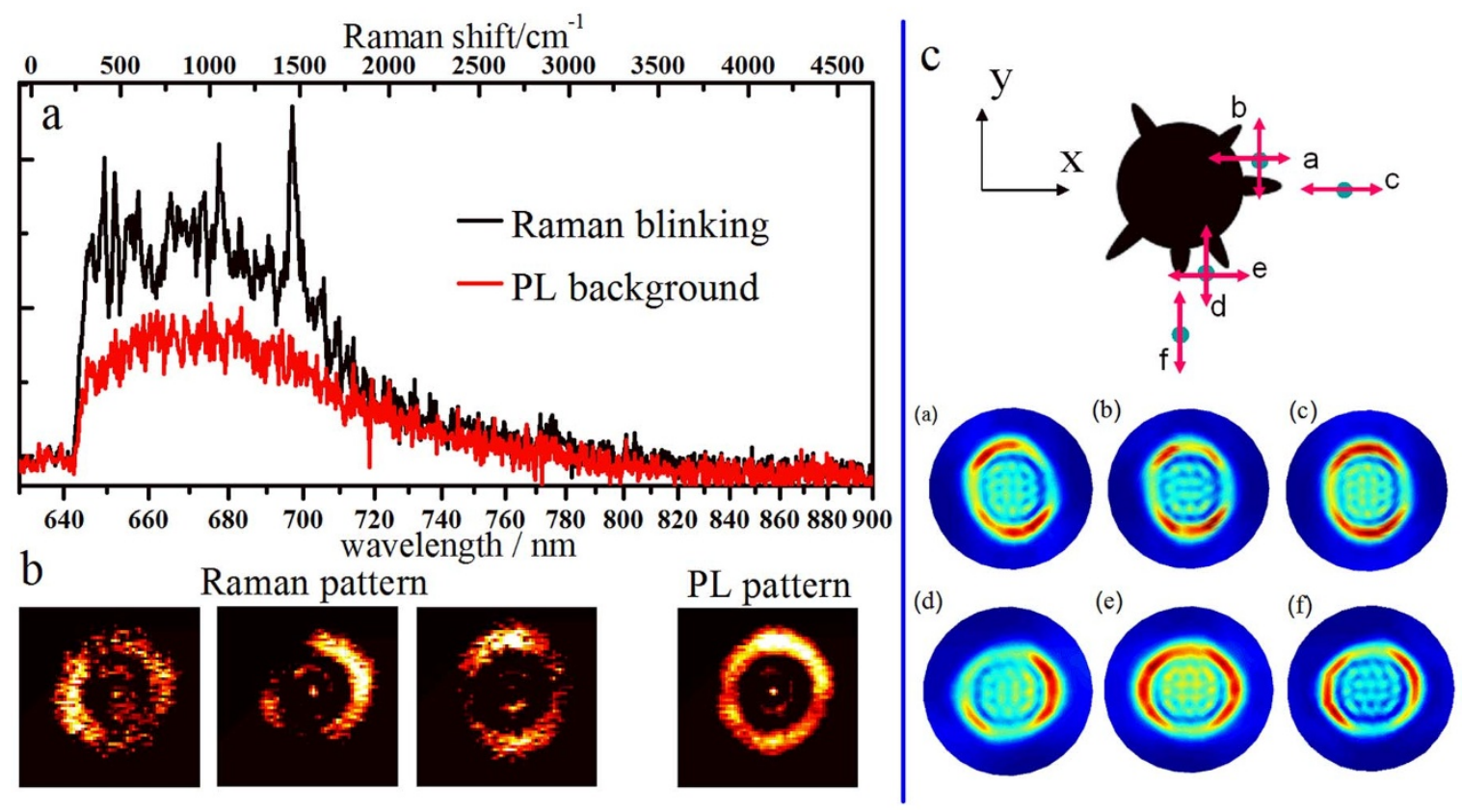

Figure $5 \mid$ (a) Raman blinking spectrum, (b) Raman imaging patterns, and the corresponding PL background spectrum and imaging pattern. Dynamic changes in the Raman blinking patterns for a single AuNF were observed, and three representative Raman patterns are presented. (c) Theoretical simulations of the emission patterns for a single dipole emitter located at various positions with various dipole orientations near a AuNF. The theoretical results qualitatively explain the changes in the Raman patterns caused by a local coupling effect with the antenna. 
bands in the PL spectra do not allow us to discriminate between the above two mechanisms ${ }^{28}$. The current results imply that the plasmon coupling in the complex nanostructures of the AuNFs plays a crucial role in such plasmon-associated PL processes. In addition, the plasmonic radiative decay channels of the AuNFs possess a relatively rapid relaxation rate, which results in an emission shape that is dependent on the initial excited states, i.e., the wavelength of the excitation source. In future studies, the polarization properties of the PL emission will be investigated together with the imaging patterns. It is also interesting to correlate the scattering patterns and the PL patterns of the same nanostructure to obtain a clearer picture of relations between the LSPRs and the PL. In addition, in the present study of PL-pattern simulations, we modeled the direction of the AuNF tips only within the $x-y$ plane, for simplicity. It should be acknowledged that the tips pointing in the $\mathrm{z}$ direction would also non-negligibly affect the emission directivity. Nevertheless, the present simulations qualitatively display the essential features of the measured PL patterns.

In conclusion, we synthesized highly branched gold AuNFs and extensively investigated the one-photon luminescence characteristics of such complex-structured nanoparticles. In contrast to nanostructures with simple shapes, the PL spectra and far-field patterns of the AuNFs exhibited distinct features, i.e., these properties presented strong dependence on the excitation wavelength and polarization. The strong coupling of surface plasmon modes associated with the core and tips results in multiple and broadband plasmon resonances. Due to the rapid damping of the LSPR modes, the excited plasmons were likely to undergo radiative decay directly from the initial excited states near the laser excitation. The PL properties were further investigated in situ by recording the angular distribution in the far field. The basic features of the PL radiation patterns were qualitatively reproduced by the theoretical calculations. Additionally, SERS blinking was observed to accompany the strong PL background in the experiments. The relationship between the Raman patterns and PL patterns was analyzed and discussed. These results provide a deeper understanding of the PL process, especially for complex metallic nanostructures, which will benefit potential applications such as optical imaging and sensing.

\section{Methods}

Sample preparation. AuNFs were synthesized following the amine-reducing method ${ }^{9}$. Briefly, an aqueous $\mathrm{HAuCl}_{4}(200 \mu \mathrm{L}, 1 \mathrm{wt} \%)$ solution was added to $20 \mathrm{~mL}$ of ultra-pure water in a $40^{\circ} \mathrm{C}$ water bath. Freshly prepared triethanolamine (TEA) solution $(400 \mu \mathrm{L}, 1 \mathrm{M})$ was then added with mild stirring, and the reaction was allowed to proceed for $60 \mathrm{~min}$. The synthesized gold products were further purified via centrifugation at 11,000 rpm for $10 \mathrm{~min}$, and the particles were then redispersed in ultra-pure water. The as-synthesized nanoflowers had very clean surfaces compared with those prepared using surfactant-assisted methods ${ }^{9}$. The AuNFs were next drop-cast onto a silane-functionalized glass coverslip. The coverslip was then gently rinsed with ultra-pure water and dried under flowing nitrogen. Most of the nanoflowers were isolated and well separated for the single-particle measurements. The identification of the same particles in the SEM images and the optical scanning images was achieved via a pattern-matching $\operatorname{method}^{49}$. The SEM characterizations were conducted using a field-emission scanning electron microscope (FE-SEM, Hitachi S-4800) with an accelerating voltage of $15 \mathrm{kV}$.

Optical measurements. A microspectroscopy system based on an inverted optical microscope (NTEGRA Spectra, NT-MDT) was developed to combine multifunctional optical measurements, which allowed us to identify single isolated nanoparticles and measure in situ the PL spectra and emission patterns of the same nanoparticle. The experimental configurations were described in detail in the previous study ${ }^{29}$. For the PL measurements performed in this study, AuNFs were excited using linearly polarized, $40 \mu \mathrm{W}, 532-\mathrm{nm}$ or $633-\mathrm{nm}$ lasers using a $100 \times \mathrm{NA}$ $=1.4$ oil immersion microscope objective. The resulting PL signal was collected using the same objective, passed through a dichroic mirror and a long-pass filter, and focused into the spectrometer. The integration time used to acquire the spectra was $10 \mathrm{~s}$. The PL and SERS patterns were imaged with a CCD camera (Princeton Instruments ProEM 512B). The sample was also characterized in situ using an AFM mounted on top of an optical microscope for morphology measurements.

Numerical simulation. Three-dimensional FDTD calculations were performed to simulate the scattering spectra of single AuNFs. For simplicity of modeling, we supposed that all the tips lay within the same plane and that the electric field of the illumination plane wave was polarized in this plane. The calculations of the far-field power flow were based on the NTFF transformation method ${ }^{40}$. A single FDTD run with a monochromatic plane-wave excitation of a certain incidence angle and polarization provided the scattered near-field data that permitted the efficient and accurate calculation of the complete far-field pattern. Therefore, our simulations here produced patterns corresponding to the PL emission with a specific radiation wavelength.

1. Schuller, J. A. et al. Plasmonics for extreme light concentration and manipulation. Nat. Mater. 9, 193-204 (2010).

2. Novotny, L. \& van Hulst, N. Antennas for light. Nat. Photonics 5, 83-90 (2011).

3. Nehl, C. L., Liao, H. W. \& Hafner, J. H. Optical properties of star-shaped gold nanoparticles. Nano Lett. 6, 683-688 (2006).

4. Dondapati, S. K. et al. Label-free Biosensing based on single gold nanostars as plasmonic transducers. ACS Nano 4, 6318-6322 (2010).

5. Hao, E., Bailey, R. C., Schatz, G. C., Hupp, J. T. \& Li, S. Y. Synthesis and optical properties of "branched" gold nanocrystals. Nano Lett. 4, 327-330 (2004).

6. Kumar, P. S., Pastoriza-Santos, I., Rodriguez-Gonzalez, B., Garcia de Abajo, F. J. \& Liz-Marzan, L. M. High-yield synthesis and optical response of gold nanostars. Nanotechnology 19, 015606 (2008).

7. Wang, H. \& Halas, N. J. Mesoscopic Au "Meatball” particles. Adv. Mater. 20 820-825 (2008).

8. Xie, J. P., Zhang, Q. B., Lee, J. Y. \& Wang, D. I. C. The synthesis of SERS-active gold nanoflower tags for in vivo applications. ACS Nano 2, 2473-2480 (2008).

9. Jiang, Y., Wu, X. J., Li, Q., Li, J. \& Xu, D. Facile synthesis of gold nanoflowers with high surface-enhanced Raman scattering activity. Nanotechnology 22, 385601 (2011).

10. Yuan, H. K. et al. Gold nanostars: surfactant-free synthesis, 3D modelling, and two-photon photoluminescence imaging. Nanotechnology 23, 075102 (2012).

11. Hrelescu, C., Sau, T. K., Rogach, A. L., Jäckel, F. \& Feldmann, J. Single gold nanostars enhance Raman scattering. Appl. Phys. Lett. 94, 153113 (2009).

12. Liang, H. Y., Li, Z. P., Wang, W. Z., Wu, Y. S. \& Xu, H. X. Highly Surfaceroughened "Flower-like" silver nanoparticles for extremely sensitive substrates of surface-enhanced Raman scattering. Adv. Mater. 21, 4614-4618 (2009).

13. Wang, W. \& Cui, H. Chitosan-luminol reduced gold nanoflowers: From one-pot synthesis to morphology-dependent SPR and chemiluminescence sensing. J. Phys. Chem. C 112, 10759-10766 (2008).

14. Sajanlal, P. R. \& Pradeep, T. Mesoflowers: A new class of highly efficient surfaceenhanced Raman active and infrared-absorbing materials. Nano Res. 2, 306-320 (2009).

15. Hao, F., Nehl, C. L., Hafner, J. H. \& Nordlander, P. Plasmon resonances of a gold nanostar. Nano Lett. 7, 729-732 (2007).

16. Shao, L. et al. Plasmonic properties of single multispiked gold nanostars: correlating modeling with experiments. Langmuir 28, 8979-8984 (2012).

17. Huang, C. et al. Gain, detuning, and radiation patterns of nanoparticle optical antennas. Phys. Rev. B 78, 155407 (2008).

18. Shegai, T. et al. A bimetallic nanoantenna for directional colour routing. Nat. Commun. 2, 481 (2011).

19. Stranahan, S. M., Titus, E. J. \& Willets, K. A. Discriminating nanoparticle dimers from higher order aggregates through wavelength-dependent SERS orientational imaging. ACS Nano 6, 1806-1813 (2012).

20. Wang, H. F. et al. In vitro and in vivo two-photon luminescence imaging of single gold nanorods. Proc. Natl. Acad. Sci. 102, 15752-15756 (2005).

21. Farrer, R. A., Butterfield, F. L., Chen, V. W. \& Fourkas, J. T. Highly efficient multiphoton-absorption-induced luminescence from gold nanoparticles. Nano Lett. 5, 1139-1142 (2005).

22. Beversluis, M., Bouhelier, A. \& Novotny, L. Continuum generation from single gold nanostructures through near-field mediated intraband transitions. Phys. Rev. B 68, 115433 (2003).

23. Dulkeith, E., Niedereichholz, T., Klar, T. A. \& Feldmann, J. Plasmon emission in photoexcited gold nanoparticles. Phys. Rev. B 70, 205424 (2004).

24. Varnavski, O., Goodson, T., Mohamed, M. \& El-Sayed, M. Femtosecond excitation dynamics in gold nanospheres and nanorods. Phys. Rev. B 72, 235405 (2005).

25. Imura, K., Nagahara, T. \& Okamoto, H. Near-field two-photon-induced photoluminescence from single gold nanorods and imaging of plasmon modes. J. Phys. Chem. B 109, 13214-13220 (2005).

26. Tcherniak, A. et al. One-photon plasmon luminescence and its application to correlation spectroscopy as a probe for rotational and translational dynamics of gold nanorods. J. Phys. Chem. C 115, 15938-15949 (2011).

27. Fang, Y. et al. Plasmon emission quantum yield of single gold nanorods as a function of aspect ratio. ACS Nano 6, 7177-7184 (2012).

28. Yorulmaz, M., Khatua, S., Zijlstra, P., Gaiduk, A. \& Orrit, M. Luminescence quantum yield of single gold nanorods. Nano Lett. 12, 4385-4391 (2012)

29. Zhang, T. et al. Single bipyramid plasmonic antenna orientation determined by direct photoluminescence pattern imaging. Adv. Opt. Mater. 1, 335-342 (2013).

30 . Wu, X. et al. High-photoluminescence-yield gold nanocubes: for cell imaging and photothermal therapy. ACS Nano 4, 113-120 (2010).

31. Tunnell, J. P. et al. Two-photon-induced photoluminescence imaging of tumors using near-infrared excited gold nanoshells. Opt. Express 16, 1590-1599 (2008). 
32. Shen, H. H., Huigao, D., Joel, K. W. Y. \& Ze, X. Plasmon-Modulated Photoluminescence of Individual Gold Nanostructures. ACS Nano 6, 10147-10155 (2012).

33. Sonnichsen, C. et al. Drastic reduction of plasmon damping in gold nanorods. Phys. Rev. Lett. 88, 077402 (2002).

34. Hanke, T. et al. Efficient nonlinear light emission of single gold optical antennas driven by few-cycle near-infrared pulses. Phys. Rev. Lett. 103, 257404 (2009).

35. Le Ru, E. C. et al. Mechanisms of spectral profile modification in surface-enhanced fluorescence. J. Phys. Chem. C 111, 16076-16079 (2007).

36. Dong, Z. C. et al. Generation of molecular hot electroluminescence by resonant nanocavity plasmons. Nat. Photonics 4, 50-54 (2010).

37. Walsh, G. F. \& Dal Negro, L. Engineering plasmon-enhanced Au light emission with planar arrays of nanoparticles. Nano Lett. 13, 786-792 (2013).

38. Li, T. et al. Three-Dimensional Orientation Sensors by Defocused Imaging of Gold Nanorods through an Ordinary Wide-Field Microscope. ACS Nano 6, 1268-1277 (2012).

39. Wackenhut, F., Virgilio Failla, A., Züchner, T., Steiner, M. \& Meixner, A. J. Threedimensional photoluminescence mapping and emission anisotropy of single gold nanorods. Appl. Phys. Lett. 100, 263102 (2012).

40. Taflove, A. \& Hagness, S. C. Computational Electrodynamics: The FiniteDifference Time-Domain Method, 3rd ed., Artech House: Norwood, MA, 2005.

41. Li, Q. et al. High Surface-Enhanced Raman Scattering Performance of Individual Gold Nanoflowers and Their Application in Live Cell Imaging. Small 9, 927-932 (2013).

42. Lombardi, J. R., Birke, R. L. \& Haran, G. Single Molecule SERS Spectral Blinking and Vibronic Coupling. J. Phys. Chem. C 115, 4540-4545 (2011).

43. Curto, A. G. et al. Unidirectional emission of a quantum dot coupled to a nanoantenna. Science 329, 930-933 (2010).

44. Pakizeh, T. \& Kall, M. Unidirectional Ultracompact Optical Nanoantennas. Nano Lett. 9, 2343-2349 (2009).

45. Zhu, W., Wang, D. \& Crozier, K. B. Direct observation of beamed Raman scattering. Nano Lett. 12, 6235-6243 (2012).

46. Mooradian, A. Photoluminescence of metals. Phys. Rev. Lett. 22, 185-187 (1969).
47. Boyd, G. T., Yu, Z. H. \& Shen, Y. R. Photoinduced luminescence from the noble metals and its enhancement on roughened surfaces. Phys. Rev. B 33, 7923-7936 (1986).

48. Shahbazyan, T. V. Theory of plasmon-enhanced metal photoluminescence. Nano Lett. 13, 194-198 (2013).

49. Chang, W. S., Ha, J. W., Slaughter, L. S. \& Link, S. Plasmonic nanorod absorbers as orientation sensors. Proc. Natl. Acad. Sci. U.S.A. 107, 2781-2786 (2010).

\section{Acknowledgments}

We thank Y. Zhu and Q.X. Lu for assistance with the experiments. This work was supported by the National Key Basic Research Program of China (grant no. 2013CB328703) and the National Natural Science Foundation of China (grant nos. 11374026, 61008026, 11121091, and 91221304).

\section{Author contributions}

G.W.L. and Q.H.G. designed the study. T.Y.Z. and G.W.L. fabricated the samples and collected and analyzed the data. T.Y.Z. and H.M.S. performed the numerical simulations. T.Y.Z., G.W.L. and Q.H.G. wrote the paper. All the authors discussed the results and commented on the manuscript.

\section{Additional information}

Competing financial interests: The authors declare no competing financial interests. How to cite this article: Zhang, T.Y. et al. Photoluminescence of a single complex plasmonic nanoparticle. Sci. Rep. 4, 3867; DOI:10.1038/srep03867 (2014). (c) (i) (2) This work is licensed under a Creative Commons Attributionvisit http://creativecommons.org/licenses/by-nc-sa/3.0 